\title{
Association between adiponectin gene T45G polymorphism and nonalcoholic fatty liver disease risk: a meta-analysis
}

\author{
W. Zhang', L.Q. Zhu', X.L. Huo', J. Qin² and G.Y. Yuan ${ }^{3}$ \\ 1'Department of Gastroenterology, Affiliated Hospital of Jiangsu University, \\ Zhenjiang, China \\ ${ }^{2}$ Department of Gastroenterology, \\ Xinjiang Production and Construction Corps-Four Division Hospital, Yining, China \\ ${ }^{3}$ Department of Endocrinology, The Affiliated Hospital of Jiangsu University, \\ Zhenjiang, China \\ Corresponding author: W. Zhang \\ E-mail: zhangwei12000@126.com \\ Genet. Mol. Res. 15 (1): gmr.15017190 \\ Received August 10, 2015 \\ Accepted October 6, 2015 \\ Published January 8, 2016 \\ DOI http://dx.doi.org/10.4238/gmr.15017190
}

\begin{abstract}
Numerous epidemiological investigations have evaluated the association between adiponectin gene T45G polymorphism and risk of nonalcoholic fatty liver disease (NAFLD). However, the results of these studies have proven to be inconsistent. Therefore, we conducted a metaanalysis to obtain a more accurate estimation of this association. Published articles were retrieved from PubMed and Web of Science databases and pooled odds ratios (ORs) with $95 \%$ confidence intervals (Cls) were calculated using fixed- or random-effect models. Five case-control studies incorporating 597 cases and 701 controls were included in this metaanalysis. No association between adiponectin gene T45G polymorphism and NAFLD was established (TT vs GG: OR $=0.83,95 \% \mathrm{Cl}=0.37-1.86$; $\mathrm{TG}$ vs GG: OR $=0.76,95 \% \mathrm{Cl}=0.33-1.79$; dominant model: $\mathrm{OR}=0.83,95 \% \mathrm{Cl}$ $=0.37-1.84$; recessive model: $\mathrm{OR}=1.10,95 \% \mathrm{Cl}=0.69-1.76)$. Moreover, in a subgroup analysis, no significant correlation was found among Asian
\end{abstract}


subjects. In conclusion, the T45G polymorphism of the adiponectin gene may not constitute an NAFLD risk factor. However, this needs to be further validated in single large well-designed future studies.

Key words: Adiponectin; Nonalcoholic fatty liver disease; Polymorphism; Meta-analysis

\section{INTRODUCTION}

With the dramatic increase in the prevalence of obesity and metabolic syndrome, nonalcoholic fatty liver disease (NAFLD) has become a major public health issue worldwide (Ahmed, 2015). NAFLD includes a spectrum of liver diseases that ranges from simple hepatic steatosis to steatohepatitis, which may subsequently progress to more severe conditions such as cirrhosis or liver cancer (Wong et al., 2011; Della Corte et al., 2012). The underlying mechanisms responsible for the development and progression of NAFLD are poorly understood. Previous studies have reported that obesity, hypoadiponectinemia, hyperinsulinemia, and diabetes may be causally related to the development of this disease (Shimabukuro et al., 2013). Moreover, there is currently increasing evidence that genetic factors play important roles in its progression (Romeo et al., 2008).

Adiponectin is a multifunctional adipocytokine secreted mainly by white adipose tissue, which performs a key function in modulating insulin sensitivity and energy metabolism (BrochuGaudreau et al., 2010). The 18.5-kb gene encoding adiponectin is located on chromosome 3q27 and consists of three exons. To date, multiple polymorphisms of this gene have been identified (Gable et al., 2006). One of the most commonly studied of these is the single nucleotide polymorphism T45G, comprising a silent $T$ to $G$ substitution in exon 2. Several studies have demonstrated that this polymorphism can affect plasma adiponectin levels (Li et al., 2007), and previous meta-analyses have shown that it may contribute to the pathogenesis of polycystic ovary syndrome (Gao et al., 2012).

A number of recent studies have investigated the association between adiponectin gene T45G polymorphism and NAFLD risk. However, rather than reaching a conclusive consensus, their results are conflicting. Such discrepant findings may be due to small sample sizes, differences in ethnicity, and other limitations in study design ( $\mathrm{Li}$ et al., 2014). Therefore, the aim of this investigation was to conduct a meta-analysis of pooled data from existing clinical studies to assess the relationship between adiponectin gene T45G polymorphism and NAFLD.

\section{MATERIAL AND METHODS}

\section{Literature search strategy}

We conducted an in silico search of the PubMed and Web of Science databases, including articles published up to June 2015, to identify studies focusing on the association between the T45G polymorphism and NAFLD risk using the following search terms: "adiponectin", "polymorphism or polymorphisms", and "nonalcoholic fatty liver disease (NAFLD)". Bibliographies of all of the papers found in this way were then checked for other relevant publications. Furthermore, we performed a manual search of the references included in pertinent articles and reviews. 


\section{Inclusion and exclusion criteria}

Potentially relevant studies were selected based on the following inclusion criteria: articles had to 1) focus on the association between the adiponectin gene T45G polymorphism and risk of NAFLD; 2) be case-control studies based on unrelated individuals; 3) provide the number of NAFLD cases and controls, and the frequency of the T45G polymorphism; and 4) be published in English. The principal reasons for excluding studies were as follows: 1) articles consisted of a case-only study or overlapping data; 2) sample size was less than 100 individuals; 3) insufficient information was available for data extraction.

\section{Data extraction}

Two investigators independently selected the papers and extracted data, and disagreements or uncertainties were resolved by consensus. The following data were obtained: first author's surname, year of publication, country of origin, ethnicity of study population, number of individuals in case and control groups, frequency of adiponectin gene T45G polymorphism in cases and controls, and consistency with Hardy-Weinberg equilibrium (HWE).

\section{Statistical analysis}

The strength of the association between the T45G polymorphism and NAFLD risk was evaluated by calculating pooled odds ratios (ORs) with $95 \%$ confidence intervals $(95 \% \mathrm{Cls})$. Pooled ORs were calculated under four comparison models: co-dominant (TT vs GG, TG vs GG, GG vs TT, TG vs TT), dominant (TTGG+TG vs GGTT), and recessive (TTGG vs TG+GGTT). The inconsistency index $\left(l^{2}\right)$ was used to estimate heterogeneity among studies, with $l^{2}>50 \%$ being considered significant. If heterogeneity was found to be present, the random-effect model (the DerSimonian-Laird method) was used to calculate pooled ORs. Otherwise, a fixed-effect model (the Mantel-Haenszel method) was employed (Higgins and Thompson, 2002). Sensitivity analysis was performed by comparing values generated under the random-effect model to those obtained with the fixed-effect model. Publication bias was determined using a funnel plot and the Begg test $(P<0.05$ was considered to represent statistically significant publication bias). Statistical analysis was performed using the Stata 12.0 software (StataCorp., College Station, TX, USA).

\section{RESULTS}

\section{Characteristics of the studies included}

To ascertain the relationship between NAFLD susceptibility and adiponectin gene T45G polymorphism, articles were retrieved based on the search criteria. The study selection process is shown in Figure 1. In total, five case-control studies including 597 cases and 701 controls were used in this meta-analysis (Musso et al., 2008; Wang et al., 2008; Tokushige et al., 2009; Zhou et al., 2010; Gupta et al., 2012). The distribution of genotypes in the control groups was found to be consistent with HWE in all studies $(P>0.05)$. The main characteristics of the eligible studies are summarized in Table 1. 


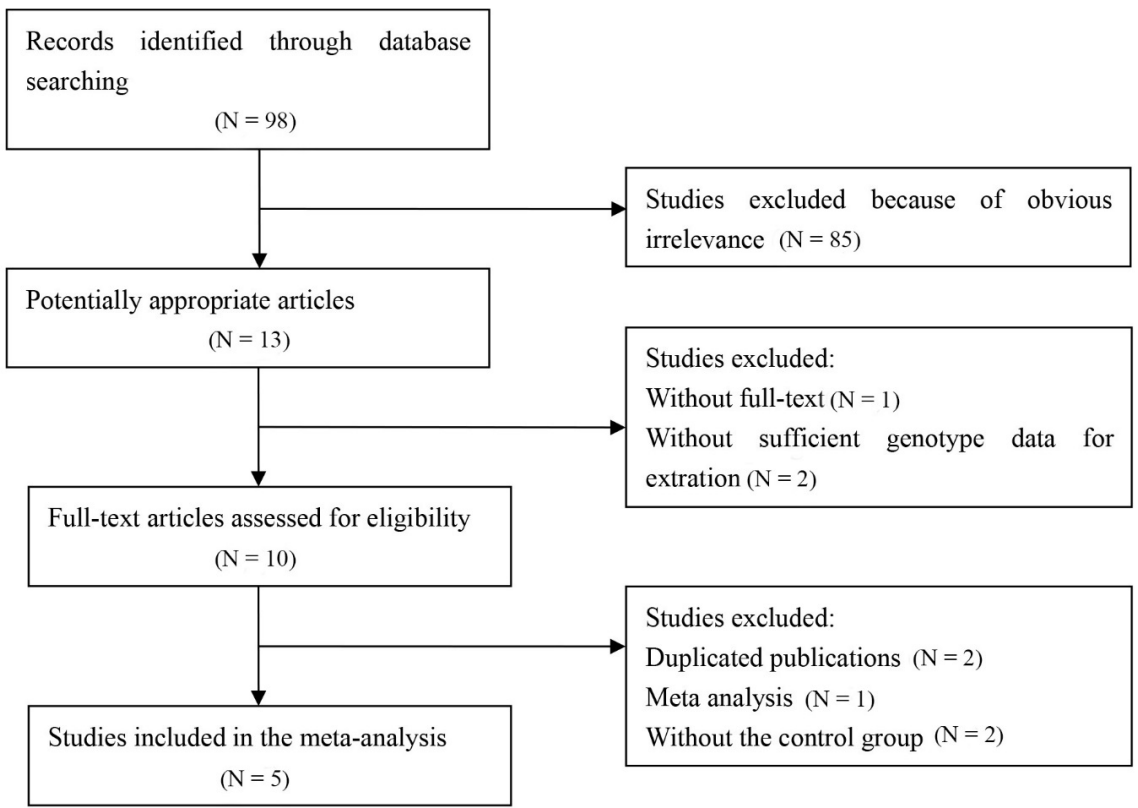

Figure 1. Flow diagram of the study search and selection process.

Table 1. Characteristics of the studies included in the meta-analysis.

\begin{tabular}{|c|c|c|c|c|c|c|c|c|c|c|}
\hline \multirow[t]{2}{*}{ Study } & \multirow[t]{2}{*}{ Country } & \multirow[t]{2}{*}{ Ethnicity } & \multirow[t]{2}{*}{ Cases/controls } & \multicolumn{3}{|c|}{ Genotypes (cases) } & \multicolumn{3}{|c|}{ Genotypes (controls) } & \multirow[t]{2}{*}{ HWE tes } \\
\hline & & & & TT & TG & GG & TT & TG & GG & \\
\hline Musso et al. (2008) & Italy & Caucasian & $70 / 70$ & 58 & 11 & 1 & 41 & 25 & 4 & 0.94 \\
\hline Wang et al. (2008) & China & Asian & $165 / 160$ & 83 & 74 & 8 & 87 & 60 & 13 & 0.56 \\
\hline Tokushige et al. (2009) & Japan & Asian & $119 / 115$ & 54 & 52 & 13 & 59 & 44 & 12 & 0.38 \\
\hline Zhou et al. (2010) & China & Asian & $106 / 106$ & 68 & 25 & 13 & 58 & 41 & 7 & 0.95 \\
\hline Gupta et al. (2012) & India & Asian & $137 / 250$ & 84 & 45 & 8 & 175 & 72 & 3 & 0.14 \\
\hline
\end{tabular}

HWE = Hardy-Weinberg equilibrium.

\section{Quantitative synthesis}

The combined results concerning the T45G polymorphism and NAFLD risk are summarized in Figure 2 and Table 2. When all eligible studies were pooled into one dataset for the meta-analysis, we found no statistical association between this polymorphism and NAFLD risk (TT vs GG: OR = $0.83,95 \% \mathrm{Cl}=0.37-1.86$; TG vs GG: OR $=0.76,95 \% \mathrm{Cl}=0.33-1.79$; dominant model: $\mathrm{OR}=0.83$, $95 \% \mathrm{Cl}=0.37-1.84$; recessive model: $\mathrm{OR}=1.10,95 \% \mathrm{Cl}=0.69-1.76)$. Sensitivity analyses were conducted by altering the statistical models. No discernible effect was noted, indicating that our results were statistically robust. In the stratified analysis by ethnicity, no association between the adiponectin gene T45G polymorphism and NAFLD risk was observed in Asian subjects (TT vs GG: $\mathrm{OR}=0.69,95 \% \mathrm{Cl}=0.32-1.48 ; \mathrm{TG}$ vs GG: OR $=0.69,95 \% \mathrm{Cl}=0.27-1.78$; dominant model: $\mathrm{OR}=$ $0.70,95 \% \mathrm{Cl}=0.31-1.58$; recessive model: $\mathrm{OR}=0.87,95 \% \mathrm{Cl}=0.69-1.10)$. 


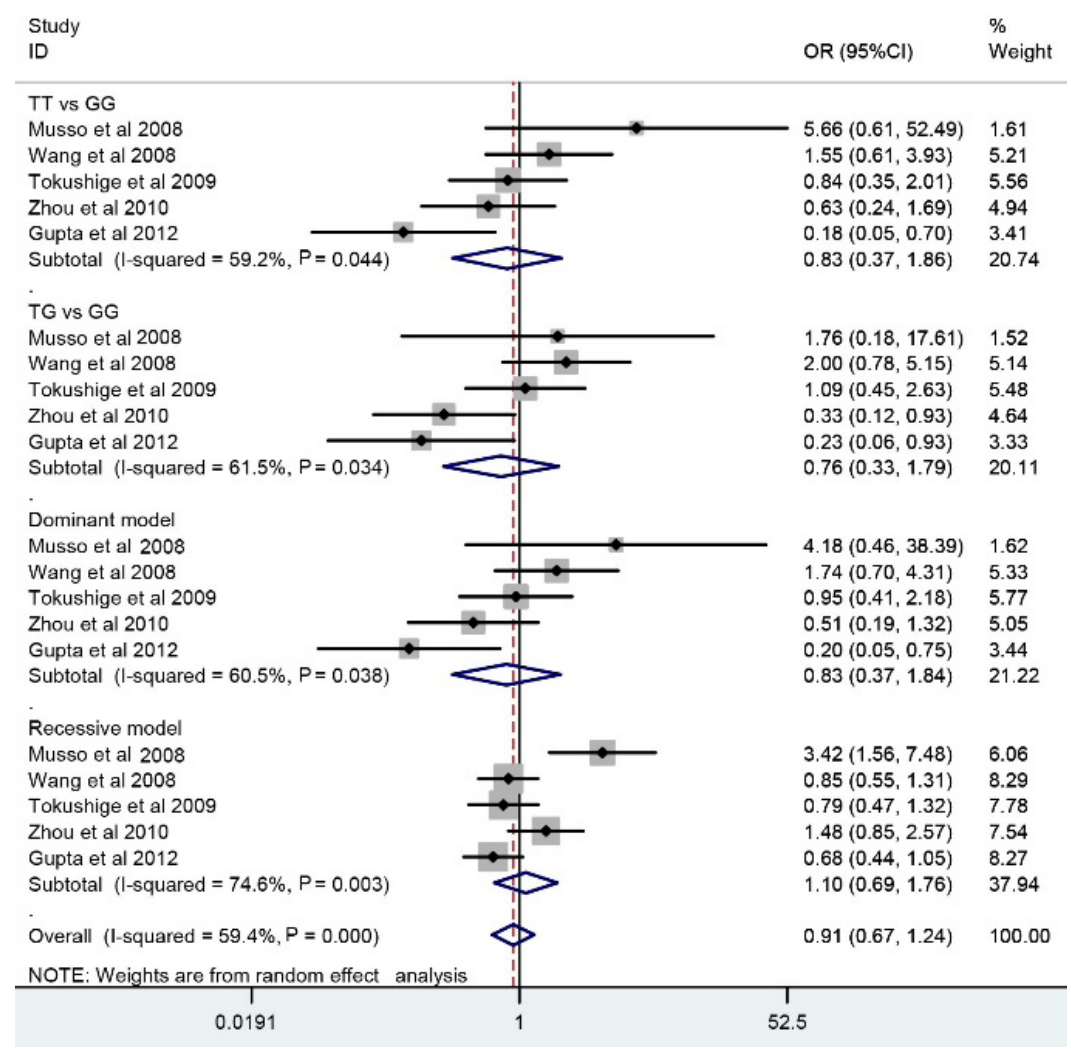

Figure 2. Meta-analysis of the relationship between adiponectin gene T45G polymorphism and nonalcoholic fatty liver disease risk. $\mathrm{OR}=$ odds ratio; $\mathrm{Cl}=$ confidence interval.

Table 2. Summary of odds ratios and $95 \%$ confidence intervals relating to the association between the adiponectin gene T45G polymorphism and nonalcoholic fatty liver disease risk.

\begin{tabular}{|c|c|c|c|c|c|c|c|}
\hline \multirow[t]{2}{*}{ Subgroup } & \multirow[t]{2}{*}{ Genetic model } & \multirow{2}{*}{$\frac{\text { Sample size }}{\text { Cases/controls }}$} & \multirow[t]{2}{*}{ Model } & \multicolumn{2}{|c|}{ Test of heterogeneity } & \multicolumn{2}{|c|}{ Test of association } \\
\hline & & & & $R(\%)$ & $\mathrm{P}$ & OR & $95 \% \mathrm{Cl}$ \\
\hline \multirow[t]{4}{*}{ Overall } & TT vs GG & $597 / 701$ & Random & 59.2 & 0.04 & 0.83 & $0.37-1.86$ \\
\hline & TG vs GG & & Random & 61.5 & 0.03 & 0.76 & $0.33-1.79$ \\
\hline & Dominant & & Random & 60.5 & 0.04 & 0.83 & $0.37-1.84$ \\
\hline & Recessive & & Random & 74.6 & 0.00 & 1.10 & $0.69-1.76$ \\
\hline \multirow[t]{4}{*}{ Asians } & TT vs GG & $527 / 631$ & Random & 56.0 & 0.08 & 0.69 & $0.32-1.48$ \\
\hline & TG vs GG & & Random & 0.0 & 0.62 & 0.69 & $0.27-1.78$ \\
\hline & Dominant & & Random & 0.0 & 0.67 & 0.70 & $0.31-1.58$ \\
\hline & Recessive & & Fixed & 0.0 & 0.42 & 0.87 & $0.69-1.10$ \\
\hline
\end{tabular}

$\mathrm{OR}=$ odds ratio $; 95 \% \mathrm{Cl}=95 \%$ confidence interval.

\section{Publication bias}

To assess publication bias in the literature used, a Begg's funnel plot was generated and its asymmetry measured (Figure 3 ). No publication bias was apparent (all $P>0.05$ ). 


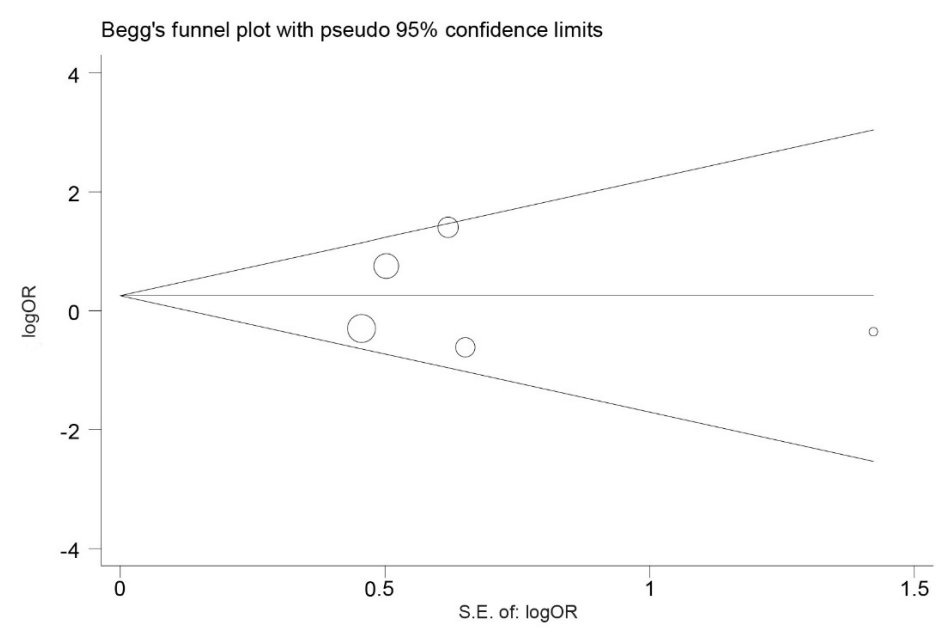

Figure 3. Funnel plot relating to adiponectin gene T45G polymorphism and susceptibility to nonalcoholic fatty liver disease. $\mathrm{OR}=$ odds ratio.

\section{DISCUSSION}

NAFLD is the most common form of chronic liver disease, and may progress to more severe conditions such as cirrhosis or liver cancer. Obesity, insulin resistance, and metabolic syndrome are the major risk factors associated with NAFLD pathophysiology. However, its pathogenesis is incompletely understood. Adiponectin, one of the most abundant cytokines, is secreted by adipose tissue, which regulates glucose and lipid metabolism (Hotta et al., 2000). Previous studies have demonstrated that a reduction in the systemic level of adiponectin has clinically significant effects and is closely associated with NAFLD (Farrell and Larter, 2006). Furthermore, prior research has shown that the T45G polymorphism can affect plasma adiponectin concentration (Li et al., 2007). Several investigations have suggested that this adiponectin sequence variation might be implicated in NAFLD pathogenesis. However, findings contradicting this proposition have also been published. Therefore, we conducted a meta-analysis of all relevant studies to comprehensively evaluate the correlation between adiponectin gene T45G polymorphism and susceptibility to NAFLD.

This is the first systematic study of the association between the T45G polymorphism and risk of this disease using a meta-analysis approach. Ultimately, five case-control studies were included and assessed, involving a total of 597 cases and 701 controls. Our results suggested that there was no significant association between the adiponectin T45G polymorphism and NAFLD risk in the overall study population. Because of inter-study environmental and genetic background differences, we performed an ethnicity-specific subgroup analysis, which also showed that this adiponectin polymorphism was not associated with NAFLD risk in Asian subjects. As only one paper involved a Caucasian population, further investigations focusing on Caucasian patients should be taken into consideration in future analysis. Owing to the limited number of eligible studies retrieved for this meta-analysis, caution should be exercised when considering any conclusions drawn.

Meta-analysis is a useful method for overcoming the disadvantages of individual studies by increasing statistical power, but this approach has some limitations. First, between-study heterogeneity in the present study was unsatisfactorily explained, despite the use of subgroup 
and sensitivity analyses to determine its source. Such heterogeneity might be due to various reasons, including variations in genotyping methods, sample sizes, and other elements of study design. Second, meta-analysis is a retrospective technique that may lead to subject selection bias, and thereby have an effect on the reliability of any results obtained. Third, gene-gene and gene-environment interactions were not tested in the present study, due to the lack of information available in the source articles.

In conclusion, our meta-analysis suggests that adiponectin gene T45G polymorphism is not associated with NAFLD. However, large-scale studies considering gene-gene and geneenvironment interactions should be conducted to further investigate this relationship.

\section{Conflicts of interest}

The authors declare no conflict of interest.

\section{ACKNOWLEDGMENTS}

Research supported by grants from the Social Development of Science and Technology Support Projects of Zhenjiang (\#SH2013036) and the Science and Technology Assistance Projects of Xinjiang Province (\#2014AB045).

\section{REFERENCES}

Ahmed M (2015). Non-alcoholic fatty liver disease in 2015. World J. Hepatol. 7: 1450-1459. http://dx.doi.org/10.4254/wjh.v7.i11.1450 Brochu-Gaudreau K, Rehfeldt C, Blouin R, Bordignon V, et al. (2010). Adiponectin action from head to toe. Endocrine 37: 1132.http://dx.doi.org/10.1007/s12020-009-9278-8

Della Corte C, Alisi A, Saccari A, De Vito R, et al. (2012). Nonalcoholic fatty liver in children and adolescents: an overview. J. Adolesc. Health 51: 305-312.http://dx.doi.org/10.1016/j.jadohealth.2012.01.010

Farrell GC and Larter CZ (2006). Nonalcoholic fatty liver disease: from steatosis to cirrhosis. Hepatology 43 (Suppl 1): S99-S112.http://dx.doi.org/10.1002/hep.20973

Gable DR, Hurel SJ and Humphries SE (2006). Adiponectin and its gene variants as risk factors for insulin resistance, the metabolic syndrome and cardiovascular disease. Atherosclerosis 188: 231-244. http://dx.doi.org/10.1016/..atherosclerosis.2006.02.010

Gao L, Zhang Y, Cui Y, Jiang Y, et al. (2012). Association of the T45G and G276T polymorphisms in the adiponectin gene with PCOS: A meta-analysis. Gynecol. Endocrinol. 28: 106-110.http://dx.doi.org/10.3109/09513590.2010.508543

Gupta AC, Misra R, Sakhuja P, Singh Y, et al. (2012). Association of adiponectin gene functional polymorphisms (-11377C/G and $+45 T / G)$ with nonalcoholic fatty liver disease. Gene 496: 63-67.http://dx.doi.org/10.1016/i.gene.2011.12.023

Higgins JP and Thompson SG (2002). Quantifying heterogeneity in a meta-analysis. Stat. Med. 21: 1539-1558. http://dx.doi.org/10.1002/sim.1186

Hotta K, Funahashi T, Arita Y, Takahashi M, et al. (2000). Plasma concentrations of a novel, adipose-specific protein, adiponectin, in type 2 diabetic patients. Arterioscler. Thromb. Vasc. Biol. 20: 1595-1599.http://dx.doi.org/10.1161/01.ATV.20.6.1595

Li L, Wang SJ, Shi K, Chen D, et al. (2014). Correlation between MTP -493G>T polymorphism and non-alcoholic fatty liver disease risk: a meta-analysis. Genet. Mol. Res. 13: 10150-10161.http://dx.doi.org/10.4238/2014.December.4.9

Li LL, Kang XL, Ran XJ, Wang Y, et al. (2007). Associations between 45T/G polymorphism of the adiponectin gene and plasma adiponectin levels with type 2 diabetes. Clin. Exp. Pharmacol. Physiol. 34: 1287-1290. http://dx.doi.org/10.1111/j.1440-1681.2007.04713.x

Musso G, Gambino R, De Michieli F, Durazzo M, et al. (2008). Adiponectin gene polymorphisms modulate acute adiponectin response to dietary fat: Possible pathogenetic role in NASH. Hepatology 47: 1167-1177.http://dx.doi.org/10.1002/hep.22142

Romeo S, Kozlitina J, Xing C, Pertsemlidis A, et al. (2008). Genetic variation in PNPLA3 confers susceptibility to nonalcoholic fatty liver disease. Nat. Genet. 40: 1461-1465.http://dx.doi.org/10.1038/ng.257

Shimabukuro M, Higa M, Yamakawa K, Masuzaki H, et al. (2013). Miglitol, $\alpha$-glycosidase inhibitor, reduces visceral fat 
accumulation and cardiovascular risk factors in subjects with the metabolic syndrome: a randomized comparable study. Int. J. Cardiol. 167: 2108-2113.http://dx.doi.org/10.1016/j.ijcard.2012.05.109

Tokushige K, Hashimoto E, Noto H, Yatsuji S, et al. (2009). Influence of adiponectin gene polymorphisms in Japanese patients with non-alcoholic fatty liver disease. J. Gastroenterol. 44: 976-982.http://dx.doi.org/10.1007/s00535-009-0085-z

Wang ZL, Xia B, Shrestha U, Jiang L, et al. (2008). Correlation between adiponectin polymorphisms and non-alcoholic fatty liver disease with or without metabolic syndrome in Chinese population. J. Endocrinol. Invest. 31: 1086-1091. http://dx.doi.org/10.1007/BF03345657

Wong VW, Wong GL, Yip GW, Lo AO, et al. (2011). Coronary artery disease and cardiovascular outcomes in patients with non-alcoholic fatty liver disease. Gut 60: 1721-1727.http://dx.doi.org/10.1136/gut.2011.242016

Zhou YJ, Li YY, Nie YQ, Yang H, et al. (2010). Influence of polygenetic polymorphisms on the susceptibility to non-alcoholic fatty liver disease of Chinese people. J. Gastroenterol. Hepatol. 25: 772-777.

http://dx.doi.org/10.1111/j.1440-1746.2009.06144.x 